\title{
Influence of Magnetic Order Parameter Symmetry and Spin Fluctuations on Forming of Ferroelectric Order in $\mathrm{Mn}_{2} \mathrm{O}_{5}$ Oxides
}

\author{
Vladimir Men`shenin ${ }^{1}$
}

${ }^{1}$ M.N. Miheev Institute of Metal Physics of Ural Branch of RAS, 620108 Yekaterinburg, Russia

\begin{abstract}
The magnetic phase transitions from paramagnetic to magnetic phases have studied in $\mathrm{RMn}_{2} \mathrm{O}_{5}$ oxides. Using a symmetry analysis and renormalization group method it was shown that an appearance of the electric polarization or lack whereof in the magnetic phases are determined the symmetry of these phases and spin fluctuations in the vicinity of the phase transitions.
\end{abstract}

The compounds were investigated intensively in the last twenty years in which the orderings of the different nature coexist. The $\mathrm{RMn}_{2} \mathrm{O}_{5}$ oxides are belonged to such systems. The long range magnetic order and ferroelectric order coexist in these oxides in the certain temperature interval. There is the strong interaction between the magnetic and ferroelectric subsystems in these compounds because the electric polarization arises only in the magnetic phase. The important difference $\mathrm{RMn}_{2} \mathrm{O}_{5}$ oxides from the manganites is that electric polarization achieves the biggest quantity in the commensurable magnetic phase whereas the polarization coexists with the long range magnetic order only in the incommensurable magnetic phase of the manganites. It was shown experimentally that electric polarization exists in the $\mathrm{RMn}_{2} \mathrm{O}_{5}(\mathrm{R}=$ rare earth, Y, Bi) compounds where the rare earth element is heavier than neodymium. The ferroelectric order appears in the one direction incommensurable magnetic phase for the compounds with the rare earth element and Y. The symmetry of the crystal lattice is not violated at low temperature when electric polarization appears since where are only nuclear Bragg peaks on the neutron diffraction patterns which have existed in the paramagnetic phase. The authors of the some papers have supposed that the additional peaks were absent due to the extremely small atomic displacements. These displacements cannot be measured experimentally.

Let us note that chemical bondings have the ioncovalent character with a significant shear charge of the electrons, which delocalize between the ions. These electrons give the essential contribution to the electric polarization. In this case we can go over from the determination of the electric polarization through the charge distribution to its definition through the variation of the electric polarization in the process of the measurement for example when the electric field is turned on. In this case, the lattice of the values for the electric polarization can include the nonzero polarization vector. Besides the space inversion is absent as the element of the exact symmetry in the incommensurable magnetic phase.

The situation is never simple if the phase transition occurs from the paramagnetic phase (PMP) to the commensurable structure. For example, the transition takes place to magnetic phase with the propagation vector $\mathbf{k}=\{1 / 2,0,1 / 2\}$ in the oxide $\mathrm{BiMn}_{2} \mathrm{O}_{5}$ and transition happens to phase with $\mathbf{k}=$ $\{1 / 2,0,0\}$ in the oxide $\mathrm{PrMn}_{2} \mathrm{O}_{5}$. The electric polarization has been found in the $\mathrm{BiMn}_{2} \mathrm{O}_{5}$ but it has been absent in the $\mathrm{PrMn}_{2} \mathrm{O}_{5}$ although the space group representations coincide for these propagation vectors. I would like to show the reasons of such behavior analyzing the magnetic phase transitions in these compounds.

Let us consider the appearance of the electric polarization for the phase transition from the PMP to the one direction incommensurable magnetic phase in the $\mathrm{RMn}_{2} \mathrm{O}_{5}$ oxides where $\mathrm{R}$ is the rare earth element. The propagation vector of the incommensurable magnetic structure is $\mathbf{k}=(1 / 2,0, v), v \neq 1 / 2$. The components of the order parameter transform under the full irreducible representation (IR) of the space group Pbam $\left(D_{2 h}^{9}\right)$. This IR is described by the matrices of fourth order. In this case, the order parameter has four components. The thermodynamic potential contains the usual terms of second and fourth order by the order parameter components, wherein a one of these terms is the product of four order parameter components, Lishitz invariant with first derivative with respect to the coordinate $\mathrm{z}$ of the order parameter components, two Dzjaloshinskii invariants and the heterogeneity exchange energy [1]. The $W_{1}, W_{2}$ values are coefficients in front of Dzjaloshinskii invariants respectively. Let us denote the order parameter components as $\left(\eta_{1}, \eta_{2}, \xi_{1}, \xi_{2}\right)$. There are the relations between these components

$$
\begin{aligned}
& \eta_{1}+\xi_{2}=\rho_{1} \exp \left(i \phi_{1}\right), \eta_{1}-\xi_{2}=\rho_{1} \exp \left(-i \phi_{1}\right), \\
& \xi_{1}-\eta_{2}=\rho_{2} \exp \left(i \phi_{2}\right), \xi_{1}+\eta_{2}=\rho_{2} \exp \left(-i \phi_{2}\right),
\end{aligned}
$$

\footnotetext{
* Corresponding author: menshenin@imp.uran.ru
} 
where values $\rho_{1}, \rho_{2}$ are the modules of the order parameter components and values $\phi_{1}, \phi_{2}$ are the phases of these components. The analysis of the thermodynamic potential leads to the following results. There is the transition to the incommensurable magnetic phase with $\varphi_{2}=k_{0} z, k_{0}=\left|\gamma_{1}\right| / \gamma_{e x}$ if $\rho_{1}, \rho_{2}$ are changing slower than phases and the equation is performed for the phases $\varphi_{1}=\varphi_{2}+(2 m+1) \pi / 2, \quad \mathrm{~m}$ is an arbitrary integer. The $\gamma_{1}$ value is a coefficient in front of Lifshitz invariance and a $\gamma_{e x}$ value is a coefficient in front of the heterogeneity exchange energy. The electric polarization $P_{y}$, which can be determined from the condition $\partial \Phi / \partial P_{y}=0$, is $P_{y}=(-1)^{m+1} \kappa \alpha_{1} \rho_{1} \rho_{2}$. In this case, the polarization depends slowly from the coordinates and is the small value in vicinity of the phase transition. The polarization is the concomitant macroscopic order parameter.

The decrease of the temperature results in the creation of the higher harmonics. In this case, we derive the nonlinear differential equations for the phases. These equations transform to the equality $\partial^{2} \varphi_{2} / \partial z^{2}=4 v \sin 4 \varphi_{2}, \quad$ where $v$ is equal $v=\left(4 W_{1}-u_{2}\right) \rho^{2} / 16 \gamma_{e x}$, if we assume that $\rho=\rho_{1}=\rho_{2}$ and $\varphi_{1}=\varphi_{2}+3 \pi / 2$. We can arrive at the conclusion on the base of the equation for the $\varphi_{2}$ value that the soliton lattice is formed in the system. The soliton lattice becomes the energetically unfavorable state under the certain conditions and the first order transition takes place to the commensurable antiferromagnetic structure. The electric polarization achieves the biggest value in this phase. The further decrease of the temperature leads to the transition to the incommensurable magnetic phase in two directions. The electric polarization is decreased significantly in this phase.

The renormalization group analysis have been performed of the transition from the PMP to the one direction incommensurable magnetic phase. We supposed that the relativistic contribution to the energy associated with the magnetic heterogeneities is small in the vicinity of this transition where magnetic fluctuations of the exchange nature are appeared. Thus, Lifshitz invariant does not play the role in this case. There are the three critical fixed points of the renormalization group transformations then the all interaction parameters are not equal zero in the approximation lineal in $\varepsilon$. The steadiness is analyzed of these points on the base of the recursive relations for the parameters of the effective Hamiltonian [2]. We consider first of all the point where the values of the $g_{i}$ parameters are

$$
g_{1}^{*}=\frac{\varepsilon}{80 K_{4}}, g_{2}^{*}=\frac{3 \varepsilon}{40 K_{4}}, g_{3}^{*}=\frac{\varepsilon}{10 K_{4}}, g_{4}^{*}=\frac{\varepsilon}{40 K_{4}}
$$

The values $\Delta g_{i}$ are the functions of the $\xi$ parameter. The system of the equations for these values have the form

$$
\begin{aligned}
& \frac{d\left(\Delta g_{i}\right)}{d \xi}=\sum_{j} A_{i j}\left(g_{1}^{*}, g_{2}^{*}, g_{3}^{*}, g_{4}^{*}\right) \Delta g_{j}+R_{i}, \\
& i=(1, . ., 4), \xi=\ln b .
\end{aligned}
$$

where $b$ is the parameter which describes the change of the spatial scale under the Kadanoff" transformations.

In this case there are two different negative real eigenvalues and two different positive real eigenvalues of the A matrix. These results imply that trajectories of the system motion in the phase space $\left(g_{1}, ., g_{4}, r\right)$ with variable parameter $\xi$, which approach arbitrary close to point found above but do not pass through this point go away from this point to infinity. However where is the trajectory passing through the fixed point for which the system returns to this point if the one shifts from the fixed point. This behavior of the system allows one to conclude that this fixed point is a stable critical point. In this point where is the second order phase transition. The analysis of the system symmetry in this point leads to the following result. The terms with the coefficients $g_{1}, g_{2}, g_{4}$ in the Hamiltonian [2] are transformed to the expression $\left(\rho_{1}^{2}+\rho_{2}^{2}\right)^{2}$ in the notation of the equality (1). These terms have the $\mathrm{O}(2)$ symmetry. The term with the $g_{3}$ coefficient does not this symmetry since the product fourth order parameter components has the orthorhombic symmetry. Hence, the system symmetry does not increase in the order point. The spin fluctuations do not happen to the disappearing of the macroscopic order parameter in the form of the electric polarization in this case.

Let us consider the situations then the magnetic transitions come about from the PMP to the commensurable structure in the $\mathrm{RMn}_{2} \mathrm{O}_{5}$ compounds. We compare the $\mathrm{BiMn}_{2} \mathrm{O}_{5}$ oxide and $\mathrm{PrMn}_{2} \mathrm{O}_{5}$ oxide. The magnetic phase transition occurs in the antiferromagnetic structure with the propagation vector $\mathbf{k}=(1 / 2,0,1 / 2)$ in the $\mathrm{BiMn}_{2} \mathrm{O}_{5}$ oxide and in the one with $\mathbf{k}=(1 / 2,0,0)$ in the $\mathrm{PrMn}_{2} \mathrm{O}_{5}$ compound. This comparison helps us to understand the influence of the spin fluctuations at the forming electric polarization in these oxides. We note first of all that the space group of the symmetry has the same irreducible representations for these propagation vectors. Where are the two representations. We have found that magnetic structure written above could be realized only for the $v_{2}$ representation. The effective Hamiltonian of the system, which allows one to analyze the critical behavior of the $\operatorname{PrMn}_{2} \mathrm{O}_{5}$ oxide in the immediate vicinity of the transition from the paramagnetic to the antiferromagnetic phase, is given by

$$
\begin{aligned}
& H_{e f f}=\int d^{d} x\left\{\frac{r}{2}\left(\varsigma_{1}^{2}(x)+\varsigma_{2}^{2}(x)\right)+\right. \\
& +u_{1}\left[\varsigma_{1}^{4}(x)+\varsigma_{2}^{4}(x)\right]+ \\
& +u_{2} \varsigma_{1}^{2}(x) \varsigma_{2}^{2}(x)+ \\
& \left.+\frac{1}{2}\left[\frac{\partial \varsigma_{1}}{\partial x_{i}} \frac{\partial \varsigma_{1}}{\partial x_{i}}+\frac{\partial \varsigma_{2}}{\partial x_{i}} \frac{\partial \varsigma_{2}}{\partial x_{i}}\right]\right\} .
\end{aligned}
$$


In this equation $\zeta_{i}(i=1,2)$ - are the order parameter components and the $r, u_{1}, u_{2}$ parameters have the same meaning as those in the expression for the thermodynamic potential that describes phase transition in Landau theory [3]. At first sight, the $\int d^{d} x \lambda P_{y}\left(\varsigma_{1}^{2}-\varsigma_{2}^{2}\right)$ term must be included at the effective Hamiltonian due to its invariance under the space group transformations. However the expression for the electric polarization is equal zero within the framework of the Landau theory for the transition in which the both order parameter components obtain. The order parameter components are equal in the absolute value for this transition. The above expression in the Hamiltonian vanishes in the case of the transition where the one order parameter component is nonzero below the Neel's temperature. The disappearing of this term happens due to spin fluctuations as we show below.

Let us introduce a parameter $s=u_{2} / u_{1}$. Where are the three fixed points of the renormalization group transformations [4] $s=0, s=2, s=6$. The point $s=2$ is the stable critical point and the points $s=0, s=6$ are unstable critical points. The transition is realized in the system with $\zeta_{1}=0, \zeta_{2} \neq 0$. The condition $u_{2}=2 u_{1}$ is satisfied in the stable critical point. An $\mathrm{O}_{2}$ symmetry arises in the system at the phase transition point. Formally, it is rather easy to understand the emergence of symmetry. The above condition implies that, instead of two fourth-order invariance, a significant invariant $\left(\zeta_{1}^{2}+\zeta_{2}^{2}\right)^{2}$ arises in the effective Hamiltonian. Then, considering the order parameter as a two-component vector we can see that the first invariant $\zeta_{1}^{2}+\zeta_{2}^{2}$ is a scalar product and, $\left(\zeta_{1}^{2}+\zeta_{2}^{2}\right)^{2}$-the square of this product [4]. It is clear, that these invariants are invariant under the rotation of twocomponent vector at an arbitrary angle. However, the expression $\int d^{d} x \lambda P_{y}\left(\varsigma_{1}^{2}-\varsigma_{2}^{2}\right)$ is not invariant under the rotations at an arbitrary angle and is equal zero in this point. Thus, the appearance of the electric polarization is suppressed of the spin fluctuations in the $\mathrm{PrMn}_{2} \mathrm{O}_{5}$ oxides.

Let us consider the $\mathrm{BiMn}_{2} \mathrm{O}_{5}$ compound. The magnetic susceptibility at low temperature increases upon the cooling and has a broad peak with two characteristic temperatures $\mathrm{T}=39 \mathrm{~K}$ and $\mathrm{T}=36 \mathrm{~K}$, indicated the occurrence of the long-range magnetic order. The existence of the second transition indicates a slight delay in the ordering of magnetic moments associated with one position of manganese relative to other. It is found [5] that the sets of the spin orientations for the positions $4 \mathrm{~h}$ $\left(\mathrm{Mn}^{3+}\right)$ and $\left(\mathrm{Mn}^{4+}\right)$ is included in the sets of the basis functions irreducible representation $v_{1}$ space group. The effective Hamiltonian that allows to describe the two experimentally observed phase transitions has the form

$$
\begin{aligned}
& H_{\text {eff }}=\int d^{d} x\left\{\left[\frac{r_{1}}{2}\left(\eta_{1}^{2}+\eta_{2}^{2}\right)+\frac{1}{2}\left(\frac{\partial \eta_{1}}{\partial x_{i}} \frac{\partial \eta_{1}}{\partial x_{i}}+\frac{\partial \eta_{2}}{\partial x_{i}} \partial \eta_{2}\right)+u_{1}\left(\eta_{1}^{4}+\eta_{2}^{4}\right)+u_{2}\left(\eta_{1}^{2} \eta_{2}^{2}\right)+\frac{1}{2 \kappa} P_{y}^{2}\right.\right. \\
& \left.+\lambda_{1} P_{y}\left(\eta_{1}^{2}-\eta_{2}^{2}\right)\right] \\
& +\left[\frac{r_{2}}{2}\left(\varsigma_{1}^{2}+\varsigma_{2}^{2}\right)+\frac{1}{2}\left(\frac{\partial \varsigma_{1}}{\partial x_{i}} \frac{\partial \varsigma_{1}}{\partial x_{i}}+\frac{\partial \varsigma_{2}}{\partial x_{i}} \frac{\partial \varsigma_{2}}{\partial x_{i}}\right)+u_{3}\left(\varsigma_{1}^{4}+\varsigma_{2}^{4}\right)+u_{4}\left(\varsigma_{1}^{2} \varsigma_{2}^{2}\right)\right. \\
& \left.\left.+\lambda_{2} P_{y}\left(s_{1}^{2}-s_{2}^{2}\right)\right]+u_{5}\left(\eta_{1}^{2} s_{1}^{2}+\eta_{2}^{2} s_{2}^{2}\right)+u_{6} \eta_{1} \eta_{2} S_{1} s_{2}+u_{7}\left(\eta_{1}^{2} s_{2}^{2}+\eta_{2}^{2} S_{1}^{2}\right)\right\}
\end{aligned}
$$

Here $\mathrm{d}=4-\varepsilon$ is the spatial dimension, $\kappa$ is the dielectric susceptibility, the last three terms describe the interaction of the order parameters for two transitions with different temperatures, the $u_{1}, \ldots, u_{7}$ parameters have the same meaning as in Landau theory. The effective Hamiltonian contains the terms including the electric polarization $P_{y}$ oriented along the y-axis of the oxide.

We believe that the fluctuations of the order parameters are significant for the first and second transitions because $r_{1} \approx r_{2}$ in this region. Let us consider the method of the renormalization group. The system has the following fixed points then the all $u_{i}$ parameters differ from zero

$$
\begin{array}{r}
x_{1}^{*}=\frac{1}{160}, x_{2}^{*}=\frac{1}{80}, x_{3}=\frac{1}{160}, x_{4}^{*}=\frac{1}{80}, x_{5}^{*}=\frac{1}{80}, x_{6}^{*}=\frac{1}{20}, x_{7}^{*}=\frac{3}{80}, \\
x_{1}^{*}=\frac{1}{160}, x_{2}^{*}=\frac{1}{80}, x_{3}^{*}=\frac{1}{160}, x_{4}^{*}=\frac{1}{80}, x_{5}^{*}=\frac{3}{80}, x_{6}^{*}=\frac{1}{20}, x_{7}^{*}=\frac{1}{80},
\end{array}
$$

where $x_{i}=u_{i} K_{4} / \varepsilon, K_{4}=1 / 8 \pi^{2}$, the value $\varepsilon$ is a small parameter. The analysis of the stable of these points leads to the following results. The first two fixed points are the unstable points of the renormalization group transformations. The first of these points has the critical surface $\left\{u_{1}, u_{5}\right\}$. The all trajectories belonging to this surface lead to stable fixed point. The second order phase transition is realized on these trajectories. Thus, the first transition occurs as second order if the trajectory associated with this transition belongs to this surface whereas the second transition is the first order. In general case, the both transitions are the first order. The $u_{6}$ parameter is the essential parameter while $u_{7}$ is the intermediate parameter. The spin fluctuations lead to the growth in the symmetry in the other fixed points where the $u_{6}$ parameter is zero and result in the disappearance of the invariances containing the electric polarization from the effective Hamiltonian in the point of the phase transitions. The above analysis implies that only the first unstable fixed point at which the $u_{6}$ parameter is significant materializes in the system.

[1] V.V. Men`shenin, JETP, 135, 236 (2009).

[2] V.V. Men'shenin, JETP, 116, 980 (2013).

[3] V.V. Men 'shenin, JETP, 120, 1019 (2015).

[4] K.G. Wilson, M. Fisher, Phys. Rev., Lett.,

28, 240(1972).

[5] V.V. Men`shenin, JETP, 122, 145 (2016). 